\title{
The Role of CYLD in Blocking Oncogenic Cell Signaling in Melanoma
}

\section{Hengning Ke, Ramin Massoumi}

Molecular Tumor Pathology, Department of Laboratory Medicine, Lund University, Skåne University Hospital, Malmö, Sweden. Email: Ramin.Massoumi@med.lu.se

Received May $30^{\text {th }}, 2013$; revised June $29^{\text {th }}, 2013$; accepted July $6^{\text {th }}, 2013$

Copyright (C) 2013 Hengning Ke, Ramin Massoumi. This is an open access article distributed under the Creative Commons Attribution License, which permits unrestricted use, distribution, and reproduction in any medium, provided the original work is properly cited.

\begin{abstract}
Dysregulation of components of the ubiqutin system has been linked to many diseases including melanoma. This is vital since the post-translational modification of different proteins via direct ubiquitin attachment is an important process for various cellular processes. CYLD is a tumor suppressor gene and deubiquitinating enzyme, which can remove polyubiquitin chains from their specific substrate and interfere with different signaling pathways. CYLD is frequently downregulated or even lost in melanoma cell lines or tissues compared to melanocytes. Down-regulation of CYLD leads to sustained oncogenic signaling that promotes melanoma progression and metastasis. In this review, we summarize the recent insights into the mechanisms which are responsible for the down-regulation of CYLD levels in melanoma and the signaling interactions of the CYLD gene product in melanoma. We argue that these recent insights into CYLD function invite the development of novel molecular strategies for melanoma prevention and treatment.
\end{abstract}

Keywords: CYLD; Deubiquitinating Enzyme; Proliferation; Cytokinesis; Metastasis

\section{Introduction}

Melanoma is the most aggressive skin cancer, with an incidence that continues to rise. Therapeutic tools against melanoma may improve objective response rates, but often fail to reduce the long-term survival rate. Mechanistic studies have shown that both genetic and epigenetic changes are involved in melanoma initiation, cell proliferation and metastasis. Among the epigenetic changes, aberrant ubiquitinating and deubiquitinating systems occupy a paramount role in regulating various pathological processes. In addition, ubiquitinating and deubiquitinating systems regulate different cellular processes such as cell cycle progression, protein degradation, receptor endocytosis, virus budding, gene transcription, and DNA damage/repair [1,2]. Covalent attachment of ubiquitin or polyubiquitin molecules to a protein substrate is catalyzed by a three enzyme cascade, which consists of the activation of ubiquitin (ubiquitination activating enzyme E1), the transfer of activated ubiquitin to the active site cysteine of ubiquitin conjugating enzyme (E2) and finally the transfer of activated ubiquitin to a lysine of the targeted protein via a ubiquitin ligase (E3), which forms an isopeptide bond. Polyubiquitination through lysine-48
(Lys-48) causes the degradation of the target protein through the proteasome, whereas polyubiquitination through lysine-63 (Lys-63) modifies the properties of the protein. The ubiquitination process is counter-regulated by a family of deubiquitinases (DUBs) [3]. These enzymes can cleave ubiquitin from ubiquitin-conjugated protein substrates, ubiquitin precursors, ubiquitin adducts, and polyubiquitin [4]. The human genome encodes approximately 90 putative DUBs and analysis of alterations in DUB expression in melanoma by in situ hybridization on tissue microarrays identified five genes (USP10, USP 11, USP22, USP48 and COPS5) that were significantly over-expressed, compared with benign nevi [5]. Out of these five genes, the expression of USP10, USP11 and USP22 was significantly higher in metastatic melanoma compared with benign nevi, and in primitive tumors, it was suggested that their expression is associated with a more aggressive and invasive phenotype [5]. USP13 [6], BAP1 [7,8], UCHL1 [9] and CYLD are other DUBs where the role of these enzymes in melanoma progression or melanocytic tumors has been highlighted. This review will confine itself to discussing the tumor suppressor function of CYLD in melanoma. 


\section{Defining CYLD Gene Product Functions}

One of the well-studied DUBs is the cylindromatosis gene (CYLD), which was originally discovered in families with multiple cylindromas, a rare benign skin disease. Linkage analysis mapped the susceptibility CYLD gene to a single locus on chromosome $16 \mathrm{q}$ in affected families [10]. Later, it was suggested that loss of heterozygosity of CYLD is associated with the development of inherited familial cylindromatosis [11].

In general, DUBs recognize specific polyubiquitin chain linkages because Lys-63-linked and linear ubiquitin structures are markedly different from Lys-48-linked ubiquitin dimers and ubiquitin tetramers; CYLD, however, is a Lys-63-specific DUB [12]. The DUB function of CYLD is through the $\mathrm{C}$-terminal domain, which encodes an ubiquitin carboxyl-terminal hydrolase ( $\mathrm{UCH})$, and deletions or mutations in this domain result in catalytically inactive CYLD. It is now established that CYLD negatively regulates multiple signaling pathways in cancer including the NF-кB, JNK, Wnt, Notch and Bcl-3 pathways (for review see $[3,13-15])$.

Several reports have established that CYLD plays a major role in melanoma by affecting fundamental changes in various cancer signaling pathways. Here, we summarize the most recent discoveries about CYLD function and propose strategies for epigenetic therapy to target the related signaling pathway in melanoma.

\section{Down-Regulation of CYLD Levels in Melanoma}

Different studies came to the same conclusion, that CYLD mRNA and protein expression is significantly downregulated in most of the melanoma cell lines and freshly isolated melanoma cells compared to normal human melanocytes [16-18]. Down-regulation of CYLD was explained by the direct recruitment of the transcriptional repressor Snaill to the CYLD promoter leading to reduced or even absent CYLD expression [16]. In contrast, melanoma cells in which Snaill expression was abrogated showed a strong up-regulation of CYLD expression [16]. Furthermore, analyzing tissue array data, CYLD expression was inversely correlated with overall and progression-free survival [16]. Down-regulation of CYLD in melanoma results in fundamental changes in their behaviors, including proliferation cytokinesis and invasion.

\section{CYLD Inhibits Proliferation of Melanoma Cells}

Cell cycle progression is tightly regulated by a number of regulators, among which cyclin D1 is an allosteric regulator of CDK4/6 and key events in G1 progression. The expression of cyclin D1 is promoted by the activation of different genes including the oncogene protein $B$-cell CLL/lymphoma 3 (Bcl-3). Bcl-3, together with the NF-кB family member $\mathrm{p} 50 / \mathrm{p} 52$, is recruited to the cyclin $\mathrm{D} 1$ promoter and initiates cyclin D1 transcription $[19,20]$. The recruitment of Bcl-3 to the cyclin D1 in keratinocytes is ubiquitin-dependent. In the absence of CYLD, UV light causes Lys-63 chain ubiquitination and the translocation of Bcl-3 from the cytoplasm into the nucleus [21]. However, in UV light-treated control keratinocytes, CYLD removes the Lys-63 polyubiquitination from $\mathrm{Bcl}-3$ and interferes with Bcl-3 nuclear translocation [21]. In melanoma patients, expression of nitric oxide synthase (iNOS) is a hallmark for poor prognosis. It was found recently that activation of Bcl-3 and p50 homodimers drive iNOS expression which further causes melanoma tumorigenesis [22].

As mentioned earlier, CYLD expression is reduced in melanoma cells. A direct consequence of CYLD repression in melanoma is the sustained Bcl-3 localization in the nucleus and the activation Cyclin D1 promoters, which results in the proliferation of melanoma cells. Restoration of melanoma cells with CYLD, blocks ubiquitination and nuclear translocation of Bcl-3 [16]. Deubiquitination of Bcl-3 also reduces the levels of cyclin D1 in melanoma cells and causes a delay in G1-S-phase transition as well as reduced cell proliferation [16]. In line with this observation, knockdown of CYLD significantly increases the proliferation activities of melanoma cell lines. This effect was mediated via Bcl-3 nuclear translocation and cyclin D1 expression [18].

In another line of study, CYLD restoration by exogenous expression in melanoma cell lines reduced cell proliferation [17]. Importantly, the self-renew ability of cancer initiating cells in melanoma cell lines was significantly reduced by CYLD expression, as demonstrated by soft agar assay [17]. In this study, it was found that the inhibition of JNK signaling by using specific JNK inhibitors prevents the colony growth of melanoma cells [17].

\section{CYLD and Cytokinesis}

In melanoma cells, EGFP-tagged CYLD is localized to the midbody during cytokinesis. More precisely, the Nterminal but not the C-terminal domain of CYLD was localized to this region. The midbody localization of CYLD caused a significant delay in cytokinesis in melanoma cells [16]. Furthermore, it was found that the delay in cytokinesis is independent of CYLD deubiquitination activity, since the catalytically inactive mutant CYLD C/S retained this ability [16]. This delay was caused by the inactivation of HDAC6, which is a tubulin-specific deacetylase. The precise role of HDAC6 in cytokinesis is not known, but it has been suggested that HDAC6 localiza- 
tion in the midbody regulates mitosis by affecting microtubule dynamics during cytokinesis [23].

Very recently, two lines of evidences highlighted the importance of HDAC6 in melanoma progression. In the first study, it was shown that acetylation-modification of cortactin (CTTN) is important for melanoma cell motility. Knockdown of HDAC6 induced a gain of CTTN protein acetylation, which further contributed to the metastasis of melanoma cells [24]. In a second study, a selective inhibitor of HDAC6 inhibited the proliferation of B16 melanoma cells and decreased tumor cell growth in vivo [25].

\section{CYLD Inhibits Melanoma Invasiveness and Metastasis}

One of the earliest steps in melanoma development includes the disruption of E-cadherin and the increased expression of N-cadherin, which facilitates cluster formation and invasion of melanoma into the dermis. Reduced E-cadherin is regulated by the transcription repressor Snaill, while Bcl-3 recruitment to the N-cadherin promoter leads to up-regulation of N-cadherin [16]. This, in turn, leads to increased tumor cell motility and invasiveness. However, re-expression of CYLD in melanoma cells inhibited $\mathrm{N}$-cadherin expression and reduced the migratory/invasive potential of cells in vitro, and less pulmonary metastasis in a murine in vivo model. These findings were correlated with the clinical findings, where both Snaill and CYLD expression in primary tumors directly correlated with progression-free survival and overall survival of the patients [16].

In another study, Loss of CYLD in melanoma induced strong JNK activation and a subsequent increase in $\beta 1$ integrin expression. Integrin family receptors play a major role in controlling melanocyte adhesion and migration [26]. As $\beta 1$-integrin expression was shown to be highly expressed in melanoma cells, over-expression of CYLD reduced the levels of $\beta 1$-integrin [17]. In this study, it was shown that JNK/AP-1 remains constitutively active in response to CYLD loss of function, which further leads to elevated levels of $\mathrm{N}$-cadherin [17]. $\beta 1$-integrin downregulation by CYLD was mediated via suppression of AP-1 signaling. This finding indicated that JNK and $\beta 1$ integrin signaling pathways function in a regulatory loop to mediate melanoma cell migration and that JNK/AP-1 and $\beta 1$-integrin signaling pathways cross-talk is negatively regulated by CYLD [17].

In contrast to the function of CYLD in inhibiting melanoma invasion and metastasis, CYLD knockdown decreased melanoma cell migration. This mechanism was found to be mediated via the activation of RAC1 through the action of CYLD [18].

\section{Future Directions}

Since CYLD plays a central role in regulating melanoma cell proliferation and migration, searching for therapeutic drugs targeting these signaling pathways seems tempting. Targeting CYLD-related signaling pathways in cancer therapeutic design can be classified into either restoration of CYLD expression or targeting CYLD-regulated pathways (Figure 1). The identification of small molecules or inhibitors targeting snail, HDAC6, integrins and Bcl-3 can lead to an increase in CYLD expression and reduced proliferation, invasive and migratory behavior of melanoma cells (Figure 1). In breast cancer cells, it was shown that a Co(III)-DNA conjugate, Co(III)-Ebox, is a potent inhibitor of Snail [27]. Recently, inhibitors of HDACs were found to successfully arrest the cell cycle and promote apoptosis of melanoma cells compared with normal melanocytes (For review see $[28,29]$ ). Aryl urea 1 is a potent selective inhibitor for HDAC6, and the treatment of B16 melanoma cells with this inhibitor blocked tumor cell growth [25]. The expression of integrins, and especially $\alpha \mathrm{v} \beta 3$-integrin, is elevated in melanoma [30]; $\alpha \mathrm{v} \beta 3$ Integrin has been shown to be a prognostic parameter for poor clinical outcome [31]. Potential therapeutic targets for anti- $\alpha \mathrm{v} \beta 3$-integrin agents can interfere with angiogenesis, tumor growth and metastases (Figure 1). MEDI522 is an antibody that is directed against the human $\alpha \mathrm{v} \beta 3$-integrin receptor. This antibody was shown to inhibit the growth of human malignant melanoma in vivo.

We believe that an agent that induces apoptosis or immunotherapy should be combined with specific small molecules for the inhibition of multiple signaling pathways in the fight against melanoma. In addition, future research will also need to develop approaches to select and divide patients into subgroups that are more likely to respond to a particular treatment. In recent years much attention has been paid to the function of DUBs in different types of cancer, including melanoma. Understanding the function of these enzymes in melanoma oncogenesis will be essential for the improvement of diagnosis and prognosis, and the design of effective therapeutics. Inhibitors or activators of selective DUBs may serve as promising tools for anti-melanoma-targeted therapy.

\section{Acknowledgements}

Research in my laboratory is supported by the Swedish Society for Medical Research, Swedish Cancer Foundation, Swedish Medical Research Council, Royal Physiographic Society in Lund, BioCARE, Cancer Foundation, SUS Research Foundations and by funding from the European Research Council (ERC), under the European Union's Seventh Framework Programme for Research and 


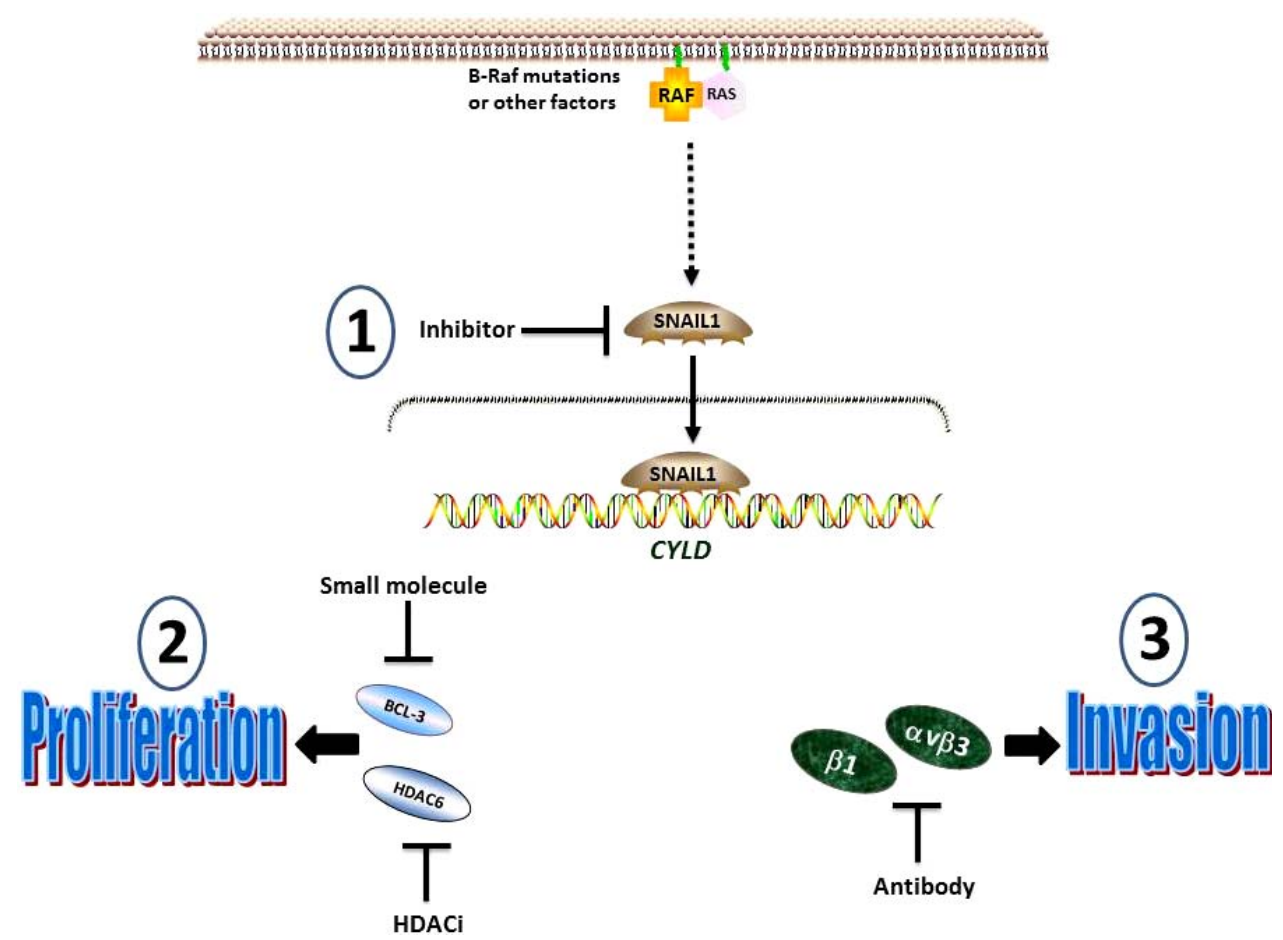

Figure 1. The identification of small molecules or inhibitors targeting snail1, HDAC6, integrins and BCL-3 can lead to reduced proliferation and invasive behavior of melanoma cells. 1) In melanoma cells, constitutively high B-Raf activation leads to high Snail1 expression which in turn causes strong suppression of CYLD. Inhibitors targeting snail allows re-expression of CYLD and reduced proliferation or migration of melanoma cells. 2) Inhibitors of HDACs (HDACi) and identification of small molecule targeting BCL-3 leads to arrest in the cell cycle and effectively blocks proliferation and tumor growth. 3) Antibodies including anti- $\alpha \mathrm{v} \beta 3$-integrin or $\beta 1$-integrin antibody can bind to the integrin receptor and inhibit the growth of human malignant melanoma by interfering with angiogenesis and metastases.

Technology Development, Grant Agreement No. [260460].

\section{REFERENCES}

[1] A. M. Weissman, "Themes and Variations on Ubiquitylation," Nature Reviews Molecular Cell Biology, Vol. 2, No. 3, 2001, pp. 169-178. doi:10.1038/35056563

[2] C. M. Pickart, "Mechanisms Underlying Ubiquitination," Annual Review of Biochemistry, Vol. 70, 2001, pp. 503533. doi:10.1146/annurev.biochem.70.1.503

[3] R. Massoumi, "Ubiquitin Chain Cleavage: CYLD at Work," Trends in Biochemical Science, Vol. 35, No. 7, 2010, pp. 392-399. doi:10.1016/j.tibs.2010.02.007

[4] S. M. B. Nijman, et al., "A Genomic and Functional Inventory of Deubiquitinating Enzymes," Cell, Vol. 123, No. 5, 2005, pp. 773-786. doi:10.1016/j.cell.2005.11.007

[5] C. Luise, et al., "An Atlas of Altered Expression of Deubiquitinating Enzymes in Human Cancer," PLoS One, Vol. 6, No. 1, 2011, Article ID: e15891. doi:10.1371/journal.pone.0015891

[6] X. Zhao, et al., "Regulation of MITF Stability by the USP13 Deubiquitinase," Nature Communications, Vol. 2, 2011, p. 414. doi: $10.1038 /$ ncomms 1421

[7] T. Wiesner, et al., "Germline Mutations in BAP1 Predispose to Melanocytic Tumors," Nature Genetics, Vol. 43,
No. 10, 2011, pp. 1018-1021. doi:10.1038/ng.910

[8] J. R. Testa, et al., "Germline BAP1 Mutations Predispose to Malignant Mesothelioma," Nature Genetics, Vol. 43, No. 10, 2011, pp. 1022-1025. doi:10.1038/ng.912

[9] J. Wulfanger, et al., "Heterogeneous Expression and Functional Relevance of the Ubiquitin Carboxyl-Terminal Hydrolase L1 in Melanoma," International Journal of Cancer, 2013. doi:10.1002/ijc. 28278

[10] P. J. Biggs, et al., "Familial Cylindromatosis (Turban Tumor Syndrome) Gene Localized to Chromosome 16Q12Q13-Evidence for Its Role as a Tumor-Suppressor Gene," Nature Genetics, Vol. 11, No. 4, 1995, pp. 441-443. doi:10.1038/ng1295-441

[11] G. R. Bignell, et al., "Identification of the Familial Cylindromatosis Tumor-Suppressor Gene," Nature Genetics, Vol. 25, No. 2, 2000, pp. 160-165. doi:10.1038/76006

[12] D. Komander, et al., "The Structure of the CYLD USP Domain Explains Its Specificity for Lys63-Linked Polyubiquitin and Reveals a B Box Module," Molecular Cell, Vol. 29, No. 4, 2008, pp. 451-464. doi:10.1016/j.molcel.2007.12.018

[13] R. Massoumi, “CYLD: A Deubiquitination Enzyme with Multiple Roles in Cancer," Future Oncology, Vol. 7, No. 2, 2011, pp. 285-297. doi:10.2217/fon.10.187

[14] R. Massoumi and R. Paus, "Cylindromatosis and the CYLD Gene: New Lessons on the Molecular Principles of Epi- 
thelial Growth Control," Bioessays, Vol. 29, No. 12, 2007, pp. 1203-1214. doi:10.1002/bies.20677

[15] K. C. Masoumi, G. Shaw-Hallgren and R. Massoumi, "Tumor Suppressor Function of CYLD in Non-Melanoma Skin Cancer," Journal of Skin Cancer, Vol. 2011, 2011, Article ID: 614097. doi:10.1155/2011/614097

[16] R. Massoumi, et al., "Down-Regulation of CYLD Expression by Snail Promotes Tumor Progression in Malignant Melanoma," Journal of Experimental Medicine, Vol. 206, No. 1, 2009, pp. 221-232. doi:10.1084/jem.20082044

[17] H. Ke, et al., "CYLD Inhibits Melanoma Growth and Progression through Suppression of the JNK/AP-1 and Beta1-Integrin Signaling Pathways," Journal of Invest Dermatology, Vol. 133, No. 1, 2013, pp. 221-229. doi:10.1038/jid.2012.253

[18] Y. Ishikawa, et al., "Down-Regulation of Cylindromatosis Gene, CYLD, Confers a Growth Advantage on Malignant Melanoma Cells While Negatively Regulating Their Migration Activity," International Journal of Oncology, Vol. 41, No. 1, 2012, pp. 53-60.

[19] P. C. Cogswell, et al., "Selective Activation of NF-Kappa B Subunits in Human Breast Cancer: Potential Roles for NF-Kappa B2/p52 and for Bcl-3," Oncogene, Vol. 19, No. 9, 2000, pp. 1123-1131. doi:10.1038/sj.onc.1203412

[20] S. G. Park, et al., "Up-Regulation of Cyclin D1 by HBx Is Mediated by NF-KappaB2/BCL3 Complex through KappaB Site of Cyclin D1 Promoter," Journal of Biological Chemistry, Vol. 281, No. 42, 2006, pp. 3177031777. doi:10.1074/jbc.M603194200

[21] R. Massoumi, et al., "CYLD Inhibits Tumor Cell Proliferation by Blocking Bcl-3-dependent NF-Kappa B Signaling," Cell, Vol. 125, No. 4, 2006, pp. 665-677. doi:10.1016/j.cell.2006.03.041

[22] D. G. Uffort, E. A. Grimm and J. A. Ellerhorst, "NF-Kappa B Mediates Mitogen-Activated Protein Kinase PathWay-Dependent iNOS Expression in Human Melanoma," Journal of Investigative Dermatology, Vol. 129, No. 1, 2009, pp. 148-154. doi:10.1038/jid.2008.205
[23] Y. Zhang, et al., "HDAC-6 Interacts with and Deacetylates Tubulin and Microtubules in Vivo," EMBO Journal, Vol. 22, No. 5, 2003, pp. 1168-1179. doi:10.1093/emboj/cdg115

[24] K. Tsunoda, et al., "Nucleus Accumbens-Associated 1 Contributes to Cortactin Deacetylation and Augments the Migration of Melanoma Cells," Journal of Invest Dermatology, Vol. 131, No. 8, 2011, pp. 1710-1719. doi:10.1038/jid.2011.110

[25] J. A. Bergman, et al., "Selective Histone Deacetylase 6 Inhibitors Bearing Substituted Urea Linkers Inhibit Melanoma Cell Growth," Journal of Medical Chemistry, Vol. 55, No. 22, 2012, pp. 9891-9899. doi:10.1021/jm301098e

[26] P. Pinon and B. Wehrle-Haller, "Integrins: Versatile Receptors Controlling Melanocyte Adhesion, Migration and Proliferation," Pigment Cell Melanoma Research, Vol. 24, No. 2, 2011, pp. 282-294. doi:10.1111/j.1755-148X.2010.00806.x

[27] A. S. Harney, T. J. Meade and C. LaBonne, "Targeted Inactivation of Snail Family EMT Regulatory Factors by a Co(III)-Ebox Conjugate," PLoS One, Vol. 7, No. 2, 2012, Article ID: e32318. doi:10.1371/journal.pone.0032318

[28] G. M. Boyle, A. C. Martyn and P. G. Parsons, "Histone Deacetylase Inhibitors and Malignant Melanoma," Pigment Cell Research, Vol. 18, No. 3, 2005, pp. 160-166. doi:10.1111/j.1600-0749.2005.00228.x

[29] M. Yoshida, et al., "Histone Deacetylase as a New Target for Cancer Chemotherapy," Cancer Chemotherapy Pharmacology, Vol. 48, No. S1, 2001, p. S20-S26. doi: $10.1007 / \mathrm{s} 002800100300$

[30] S. M. Albelda, et al., "Integrin Distribution in Malignant Melanoma: Association of the Beta 3 Subunit with Tumor Progression," Cancer Research, Vol. 50, No. 20, 1990, pp. 6757-6764.

[31] E. H. Danen, et al., "Integrins and Melanoma Progression," Recent Results Cancer Research, Vol. 128, 1993, pp. 119-132. doi:10.1007/978-3-642-84881-0 9 


\section{Abbreviations and Acronyms}

AP-1: Activator Protein 1

BAP1: BRCA1 Associated Protein-1

Bcl-3: B-cell CLL/lymphoma 3

CDK4/6: Cyclin-Dependent Kinase4/6

COPS5: COP9 constitutive photomorphogenic homolog subunit 5

CYLD: Cylindromatosis gene

CTTN: Cortactin

DUB: Deubiquitinating enzyme

HDAC6: Histone Deacetylase 6

iNOS: nitric oxide synthase

JNK: c-Jun N-terminal kinase

Lys-48: Lysine-48

Lys-63: Lysine-63
NF-кB: Nuclear Factor Kappa-light-chain-enhancer of activated $B$ cells

Notch: Notch homolog 1 translocation-associated

p50: NF-кB-p50

p52: NF-кB-p52

RAC1: Ras-Related C3 Botulinum Toxin Substrate 1

Snail1: Snail homolog 1

UCHL1: Ubiquitin Carboxyl-Terminal Hydrolase L1

USP10: Ubiquitin Specific Peptidase 10

USP11: Ubiquitin Specific Peptidase 11

USP13: Ubiquitin Specific Peptidase 13

USP22: Ubiquitin Specific Peptidase 22

USP48: Ubiquitin Specific Peptidase 48

Wnt: Wingless-Type MMTV Integration Site 